\title{
Remarks on the Reversible Asymmetry in the Opercula of the Polychæte Hydroides.
}

\author{
By
}

Yô K. Okada,

Imperial University of Kyoto, Japan.

With 5 Figures in the Text.

THE members of the family Serpulidæ are the most highly differentiated and most truly tubicolous Polychætes. They are not large as a rule, and live in a calcareous tube or in a spirally wound shell, which they build outside the body. For this manner of living one of the gill filaments, either right or left or rarely both of them, develop into a sort of plug, the operculum, that closes the opening of the tube when the animal retreats into it. C. Zeleny first showed in Hydroides dianthus (1901) and in other allied species of Polychætes (1905), that if the operculum is cut off, the small rudiment on the opposite side begins to grow, and in the course of a few weeks becomes as large as the one removed and acts as a functional organ, while from the stump of the original operculum a new small rudiment develops. An animal with a right-handed operculum is thus easily transformed into one with a left-handed one, and this can be brought back to the original right-handed form.

When the small rudimentary operculum is removed, the large functional one remains unchanged. However, if both the functional and the rudimentary opercula are taken off at the same time, the original rudimentary one may sometimes be the first to regenerate and after several days is distinctly larger than the one regenerated on the opposite side ; compensatory regulation takes place here also. That the presence of a functional organ holds its mate in check is evident. The nature of this influence is a matter for investigation.

Compensatory regulation takes place also in the asymmetrical development of the claws of the Crustacean Alpheus (H. Przibram, 1901). E. B. Wilson (1903) found that after removal of the large chela, if the nerve of the rudimentary small one be cut at the base, "the reversal in some cases at least does not take place, or is incomplete."

One of Zeleny's experiments in which the Polychrte was cut in the thoracic region, with the resulting production of double functional 
opercula, is especially interesting in connection with the explanation that the nervous system controls the reversal of asymmetry. The failure to establish the original asymmetry is here suggested to be due to the absence or to the undeveloped state of the cerebral ganglia while opercula are forming.

In Hydroides norvegica of the Plymouth district with which I have experimented, the opercula regenerated from a transverse cut of the thoracic region are rather rarely symmetrical, while in the majority of . cases one rudiment is, from the first, always larger or develops more quickly than that of the opposite side, and it alone gives rise to a functional organ. I have got only a few specimens of double opercula out of each 50 cuts, three times repeated, while specimens with two fully developed opercula on the right and the left side can be easily obtained, as will be shown later, when the growing tip of the rudimentary operculum is injured after removal of the functional one (see Fig. 3). The asymmetrical development of the opercula seems, therefore, to be more deeply impressed in the organisation of Hydroides norvegica than we have learned from Zeleny's experiments on Hydroides dianthus and other allied species, but this condition of asymmetry is very unstable, and is easily reversible by an external influence; there is only a tendency in the Polychæta for the opercula to show right-handed development. The origin of the asymmetry is due to the growth difference between two sides in the same kind of organ. Actually most of my specimens, from which a part of the thoracic segments had been removed, produced first two almost symmetrical rudiments of opercula, but one of them, generally that of the right side, grew more quickly than that of the other side, and it alone, as mentioned above, gave rise to a functional organ, possibly suppressing the development of its mate.

Zeleny also cut the anterior part of the Polychæte in question longitudinally into approximately equally right and left halves and then took off one side. A few of them are said to have showed clear structures which corresponded very well with young regenerating branchial circlets, but none lived long enough to allow of a full development of the new organs.

According to my own experience, one side of the anterior segments including the head can be cut off (obliquely, of course) almost without impeding the regenerative power of the animal. In such an operation, if the brain is left attached to the side bearing the rudimentary operculum, the latter grows and becomes a functional organ as in the case where the functional operculum only is removed; the growth of the rudimentary operculum takes place even before any other organ is regenerated from the cut surface. On the other hand, if the brain is taken off with the functional operculum, growth does not occur in the rudimentary organ. Neverthe- 
less, growth of the latter does take place as soon as the missing half of the head segment is regenerated.

As a result of his study on the "Scherenumkehr" of the decapod Crustacea, H. Przibram (1907) definitely questions whether this phenomenon of compensatory regulation is dependent on a nervous reflex set in motion by the stimulation of damaged nerves in the functional organ. According to him the nervous stimulus here plays only a secondary role, its influence being at most "Wachstumhemmung " in the developing organs.

It has already been mentioned that the presence of a functional organ in Hydroides holds the development of its mate in check. When the functional operculum is removed growth is, therefore, induced in the rudimentary one, while removal of the latter has no apparent effect on the former. When both are operated on at the same time, there will be found in some cases a reversal of asymmetry, but in other cases two functional opercula are developed, one on each side.

Since removal of the rudimentary operculum has no specific influence either on the opposite functional organ or on its own regeneration, Zeleny considers that the differences of level of the cut have no influence upon the character of the result. The condition is, however, different in the functional operculum. According to his experimental facts the most distal and the most proximal cuts agree in giving rise to a reversal of the original asymmetry, while cuts in the intermediate levels give rise in a majority of the cases to two functional opercula. Zeleny attributes this difference in the results to differences in the regeneratory development of the old functional operculum. And as to what factor or factors hold it in the rudimentary condition in some cases, while in others it is allowed to develop into a full-sized functional organ, he suggests, first, the influence of the position of the cut upon the initial stages of regeneration, and, second, the possibility of a retarding influence emanating from the new functional organ which is formed on the opposite side from the originally rudimentary one. Zeleny states that the most distal cuts do not affect the embryonic tissue at the base of the functional operculum as quickly as do the more proximal cuts, while the cuts too near the base directly injure the tissue in question, and for either reason the tissue will have had only a small start when the opposite rudimentary organ has already a considerable size. After cuts at the intermediate levels the regenerated bud on the old functional side shows greater development than after the most distal or proximal cuts, and assuming the uniform development of the bud of the rudimentary side in all cases, it has attained a considerable size at the time when the latter begins to grow. "Therefore, in the first cases, the result of simultaneous operation on both opercula is a reversal of the original condition, while in the second, it is the production of two functional opercula." 
Zeleny considers the tissue forming the base of the functional operculum in the region below the breaking joint to be embryonic. In Hydroides norvegica the connective tissue of the corresponding region is, however, not observed to be particularly different from that of the other parts. The cells of the ectodermal layer are, indeed, taller and visibly more crowded together than elsewhere, but the nuclei have a similar size and the cytoplasm shows the same appearance as in other parts (see Fig. 1, $r b, l b)$. With only such a simple fact as the crowding of the cells in the ectoderm, it is rather difficult to say that the basal part of the functional operculum, as Zeleny claims, is embryonic. On the other hand, the

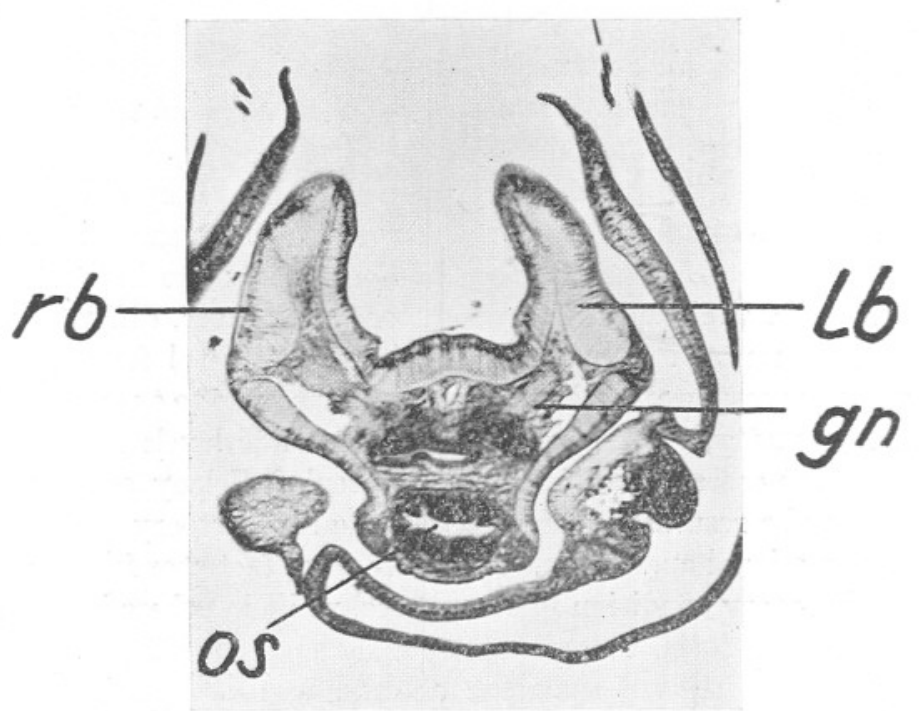

Fra. 1.-Transverse section through head of Hydroides norvegica, $r b$ and $l b$ indicating basal parts of right and left gills.

rudimentary operculum is everywhere distinctly embryonic, the cells being plump and having large nuclei, and being deeply stainable with dyes. It is somewhat club-shaped and when later it develops into a structure of two cups, it is first constricted at the level of the distal third of its length, and this distal part is further subdivided into two with accompanying growth; thus from the basal section arises the shaft, and from the middle and the distal section the lower and the upper cup of the functional operculum respectively.

When the functional operculum is injured the rudimentary organ on the opposite side is ready to start development. From 20 to 30 hours after the operation the latter has already a fairly large size and shows its three 
sections distinctly. At this stage cutting within the limit of the distal section is followed almost invariably by regeneration of the missing part, while below this level the power of regeneration decreases towards the base, and there is almost no regeneration after cuts of the basal section. The assumption of uniform regeneration of the rudimentary operculum following cuts at different levels is, therefore, challenged. In regeneration from the basal section, the future shaft is generally imperfect. When this part of the rudimentary operculum is cut after removal of the functional one, there is sometimes no reversal of the original asymmetry, since an imperfect organ practically cannot inhibit the regenerative development of the stump of the old functional operculum, or in other cases the

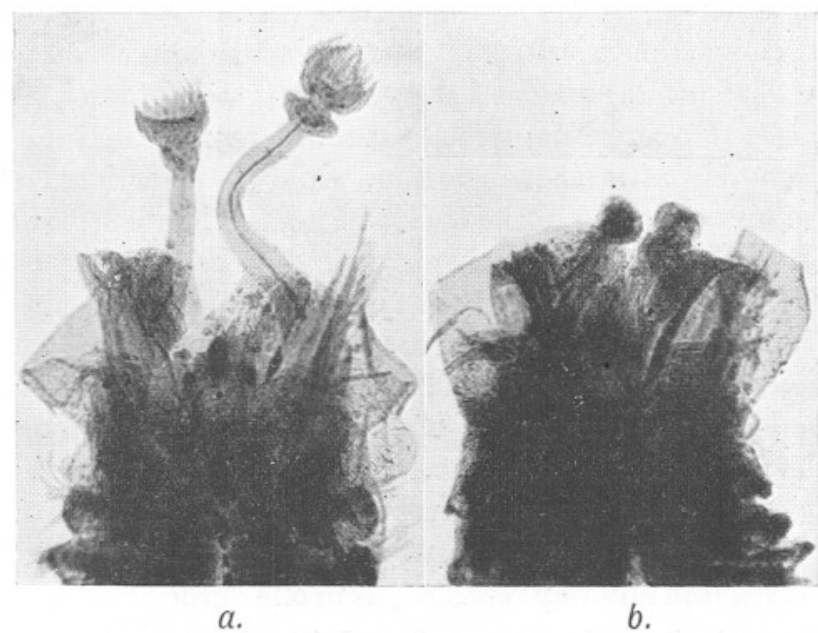

FIG. 2.-Artificial induction of symmetrical opercula, $(a)$ both functional, $(b)$ both rudimentary.

wounded shaft of the latter does not drop off. In this case perhaps an equilibrium can in some way be attained between the imperfect structures on each side. If, however, either one is taken off from the base, the equilibrium is broken, and a new rudiment is soon formed from its stump.

In a similar experiment where the rudimentary operculum was severed within the limit of the distal section, there was as a rule a reversal of asymmetry, because a prompt regeneration was still possible in the operated organ before a new rudiment makes its appearance on the opposite side. If, at the time of the operation, the functional operculum was removed from its base, two small opercula, one on each side, were formed, and in some cases both gave rise to functional organs (Fig. 2a), or in other cases both remain rudimentary (Fig. 2b). In the first case one may suppose that the new rudiment from the stump of the removed 
functional operculum has already advanced to a certain degree of development, while regeneration is going on in the operated rudimentary one, and thus it can continue to grow in spite of the presence of an inhibitory agent on the opposite side. On the other hand, if the inhibitory influence of each organ acts too strongly and checks the development of the other rudiment, the second case would result. In either case, whether inhibition takes place or not, its effect on the growth of an organ on the opposite side should be equal, otherwise one rudiment will develop more than the other.

The fact that the functional operculum has no regenerative capacity, while the rudimentary one has, leads us also to suppose that the former has used up something which the latter still retains for use in development. The difference in the results, after simultaneous operation on both functional and rudimentary opercula, may be explainable on an assumption, somewhat different from Zeleny's interpretation cited above, that the rudimentary operculum, which remained more embryonic, regenerated immediately after the operation, while the functional operculum which used up something in the course of its development, required time to regain this something, or in other words, to get back again to its original state of development. There must exist a difference of time in the regeneration of the opercula of the two sides, and according to the nature of the organ in question, the larger or well developed always checks the growth of the smaller or less developed. As a result of this antagonistic action only the larger gives rise to a functional organ, while the smaller on the opposite side is forced to remain rudimentary. When the growth of the originally rudimentary operculum, which is larger after the removal of the functional one, is in any way retarded, as in one of the above experiments, and shows no great difference from the growth-state of the new rudiment from the old functional operculum, specimens with two functional opercula may easily be produced, Figure $3 d$ being one of such productions.

T. H. Morgan (1923 and '24) has comparatively recently investigated the problem of the asymmetrical development in the chelæ of the male fiddler crab, Gelasimus pugnax, in which a reversal of asymmetry does not occur. According to him, the young male crabs have two claws, one as large as the other, and both are a little larger than those of the female of the same age, and show the swollen shape of the male large claw. At some time most of the young males lose one of the claws and produce at the next moult, in place of the lost one, a small claw of the female type. This relation, once attained, becomes fixed and even in young crabs no reversal after the removal of the large claw is possible. If young male crabs with two large claws are carefully protected from injury, they usually retain both claws throughout successive moults, and develop into a symmetrical male with two large claws. On the other hand, if both 
claws are removed from young males, at the next moult two small female claws develop, and this condition of symmetry is also retained in the successive moult. Thus an accident determines the first asymmetry of the chelæ in male Gelasimus, and it is not due to any preformative
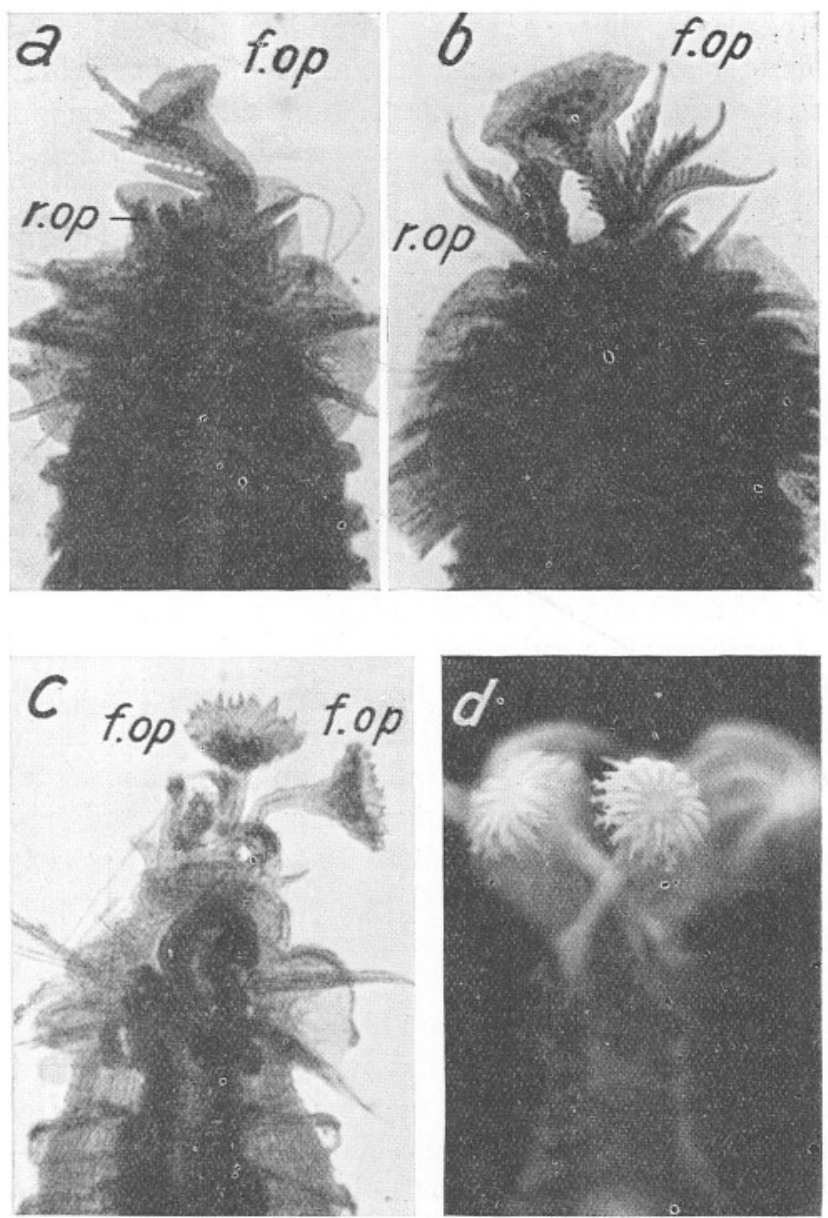

FIG. 3.- $(a)-(c)$ Anterior regenerations from thoracic segments, (d) di-opercular specimen artificially produced.

character. The asymmetry may even be introduced in this case relatively late in development, but the relation, once attained, becomes fixed and no further reversibility is possible.

As to the origin of the asymmetrical development of the opercula in Polychætes, we are much tempted to refer it also to an accidental cause, but it should not be forgotten that there is no critical period of fixation 
as in the case of the male fiddler crab. Without doubt an injury of the functional operculum is due to accident, and this external cause brings a reversal of asymmetry. But how does this external influence result in the growth of the rudimentary organ on the opposite side of the body? We know many analogous phenomena in other animal structures as well as in those of plants, but we have as yet no adequate explanation of them. The only thing we can say here is that the mechanism of this compensatory regulation is not due to a nervous reflex set up in the wounded organ, since, for example, the influence depends to a certain extent upon degrees of injury.

Zeleny (1905) has already observed that a removal of the upper cup of the functional operculum does not as a rule cause a reversal of asymmetry, while an injury below this part to the main portion of the operculum or to its stalk always does. This fact makes us doubt whether there is any special localisation of the inhibitory influence in the rudimentary organ, and leads us to suppose that if such special localisation does exist, it will be found in some part of the functional opereulum. It may be mentioned in passing that growth of the rudimentary operculum does not take place in an animal with a perfect functional operculum, even when it is drawn out of the tube and most of the gill filaments are cut off.

Before describing the results of my own experiments, a brief account is necessary of the structure of the functional operculum, which consists of a distal crown and a long proximal shaft. The former, or the operculum in the strict sense, is made up of basally, a cup corresponding to the operculum of Serpula, from the centre of which arises a second cup, which is larger than the lower and carries about 10 to 20 spines sharply pointed towards the ends. These spines radiate in all directions from the centre to the periphery, and each in Hydroides norvegica is more or less dentated (Fig. 4).

The operculum is nothing but a gill filament functionally modified. Therefore, as in the latter, immediately beneath the cuticle there lies a tall epithelium and below this a marrow-like reticulum of connective tissue, which contains a large axial blood vessel (see Fig. 4, b-d). The blood vessel coming up the shaft to the base of the lower cup and giving off ramifications to the peripheral structures of the latter, proceeds further upwards to enter the upper cup, passing through the narrow isthmus that forms the centre of the circlet of the distal spines. Thus each spine receives a direct supply of blood, which is of greenish colour in the fresh condition, and its distribution through the operculum can be easily traced in the living specimen. The distal half of the spines in the upper cup and the leaf-like processes in the lower cup have, however, no direct supply of blood.

With this preparatory knowledge of the structure of the operculum, in 


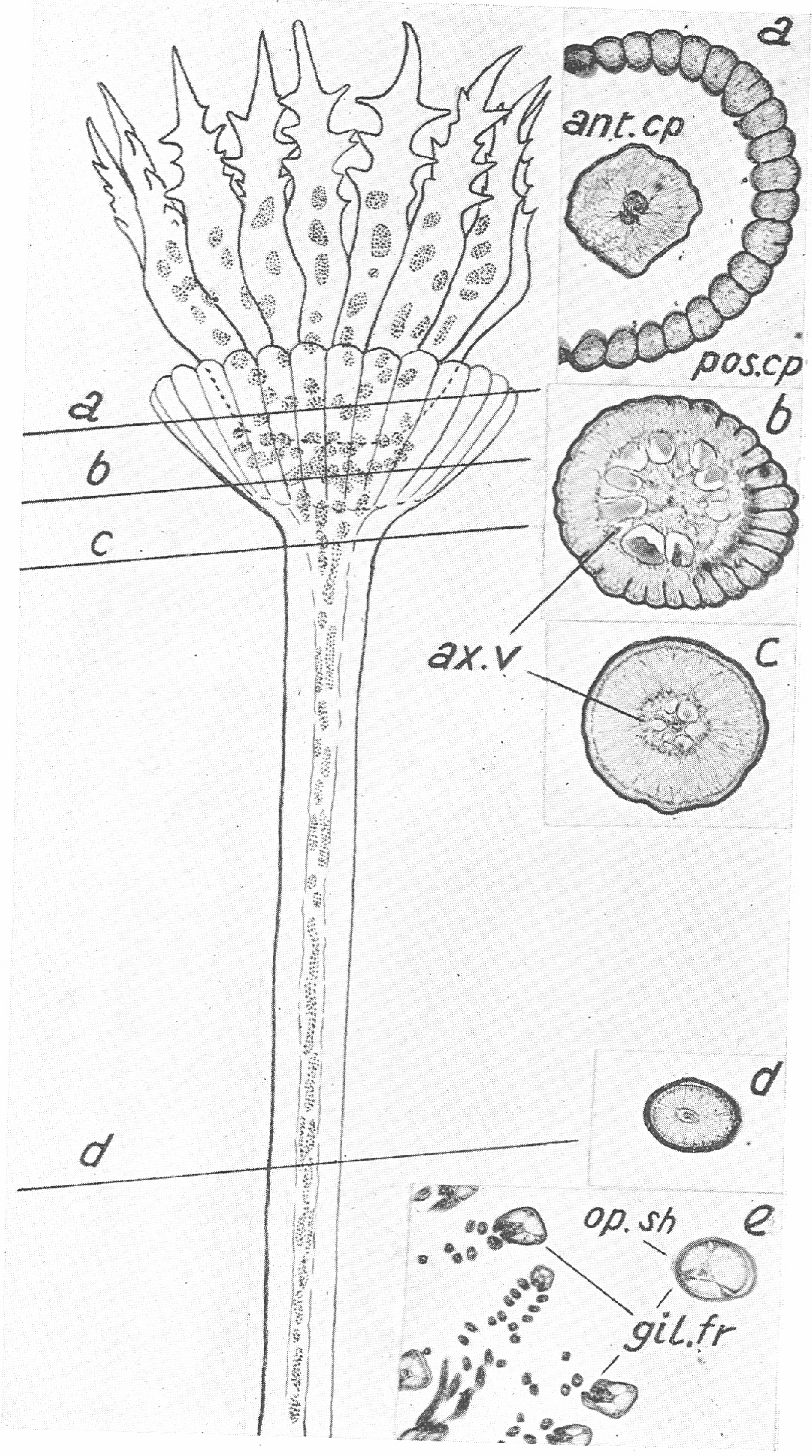

Fig. 4.-Functional operculum and its regional structural differences in transverse sections. 
the first series of my experiments the entire eirclet of the distal spines and a half of the proximal cup were removed, but the other half of the latter and the entire shaft remained intact. As a result compensatory growth took place in the rudimentary organ, which developed at the end of two weeks into a fully functional organ.

The inhibitory influence of the functional operculum must be found, therefore, outside the limit of the shaft, since in spite of its presence growth takes place in the rudimentary organ.

Next, one side of the distal as well as the proximal cup was cut off in varying degrees, but always more than half of each cup remained intact. In all cases, without exception, the rudimentary operculum grew as in the preceding experiment and developed into a functional organ. Here also it is evident that inhibitory influence of the functional operculum is not localised either in the proximal or in the distal cup, but it seems to depend on the presence of both or either one of the cups as a complete structure.

We may now ask whether or not the inhibition functions when one only of two cups, either the upper or the lower, is perfect, or whether it requires the presence of both of them. To answer this question I removed the distal circlet of spines carefully, while retaining the proximal cup intact. In that case the rudimentary operculum on the opposite side grew more slowly, compared with those of the preceding experiments, butit developed into a functional operculum in the end. The same result was obtained when one side of the proximal cup was operated on, while the circlet of distal spines remained intact. Hence we are forced to conclude that the presence of both complete cups is necessary to check the development of the rudimentary operculum.

But it was sometimes found that an imperfect removal of the spines in the distal cup or the leaf-like processes in the proximal cup did not cause a reversal of asymmetry. After repeated experiments it was found that cutting off the spines of the distal cup or taking off the leaf-like processes of the proximal cup, even all of them, but outside the limit of the direct supply of blood, had no effect, while an injury of either cup deep enough to cause an outflow of blood, induced almost invariably a growth of the rudimentary organ. Therefore, it is not necessary to take off any part of the functional operculum, but is sufficient to make a deep cut to the inner part. From these experiments it may be said that the inhibitory influence of the functional operculum depends upon the intactness of its internal constitution. Those peripheral parts such as the spines of the distal cup or the leaf-like processes of the proximal cup can be taken off, without any effect on the present phenomenon of a reversible asymmetry. Here it should be added that such a simple injury as puncturing with a fine needle, however deeply it may go, has no 
effect. But when the inner structure of the operculum is destroyed by some injury to such an extent that a greenish fluid, namely the blood, flows out, growth ensues in the rudimentary operculum.

Since cutting off the peripheral structures or a simple puncturing of the functional operculum has no effect, while deeper injuries accompanied by an outflow of blood have such an effect, we may well suppose that the phenomenon in question might be due to a circulatory disturbance. It cannot however be due to loss of blood, since on cutting off all the gill filaments or other parts of the body except the functional operculum, with greater loss of blood, there was no case in which growth took place in the rudimentary organ. It seems more plausible, therefore, to suppose a production of some substance in the wounded part of the functional operculum, and that this substance either stimulates the rudimentary organ to development or destroys the inhibitory influence of its own side, with secondarily resulting growth of the other side.

Therefore, the next step in the experiments was first to ligature the functional operculum at a given position of the shaft with a fine baby's hair and then to injure it in the more distal part, in order to see whether or not ligation stops a circulation of such a presumptive substance. The result of this experiment was entirely contrary to what was expected, and in all specimens without exception the small rudimentary operculum developed into a functional organ in due course. In the control experiment, in which the shaft of the operculum was simply ligated without subsequent injury of the distal part, the same degree of growth was also found in the rudimentary organ.

Ligation may indeed stop the circulation of blood and the distribution of material from the distal part of the operated operculum to the other parts of the body, but from the control experiment it is doubtful whether the same material might also be produced in the ligated part of the shaft in consequence of the strong constriction. I therefore abandoned this method of experiment, and injected the substance squeezed out of the wounded operculum into the body of another individual having an intact operculum. For this purpose I had to pull out 20 large animals from their calcareous tubes and to injure the tip of the functional operculum. The rudimentary organ on the opposite side was thus made to start growing. After well confirming the growth of the latter, the inner tissue of the severed operculum (two days after the operation) was pressed out with the back of a dissection knife into a clean watch glass. The substance thus obtained from 20 animals was then ground down and passed through fine meshed silk. After making double the original volume by adding filtered clean sea-water, the preparation was injected into 10 sound individuals by means of a very fine glass pipette through the biggest thoracic vein. The operated animals lived very well, but none of them 
showed any sign of a reversal of the original condition of asymmetry. Moreover there was no change either in the functional or in the rudimentary operculum to the last, even after waiting more than two weeks. Thus we have only negative results for proving the production of a growth stimulating substance in the injured operculum.

On the other hand there is no significant localisation in the functional operculum of the inhibitory influence upon the growth of the rudimentary one, while the influence most evidently depends upon the presence of a complete organisation of the former or at least upon the intactness of its internal constitution. This organ of inhibition has no regenerative capacity, as has been well demonstrated by the preceding experiments. When it is cut or injured, the part remaining is thrown off sooner or later from the body, separating by a breaking joint at the base, and a new start repeats from the beginning the original development. Generally the old shaft remains attached to the body, and the rudimentary organ begins to grow. The growth takes place only when the functional operculum is injured, but never even when all gill filaments of that side are cut off. Therefore, it is not plausible that a certain substance, even admitting such a production in the wounded place, particularly stimulates. the rudimentary organ to development, since the same substance would also be produced in the severed gill filaments as in the operated operculum. In the present case, however, it is quite certain that the wound has its. direct effect upon the inhibitory influence of the functional operculum itself. Yet it still remains to be shown whether this action is due to the production of a certain substance in the wounded tissue of the operculum. At any rate, as soon as the inhibitory influence of the functional operculum is destroyed, the rudimentary organ starts to grow and in the absence of the former controls the whole system, restricting the development of the new rudiment from the stump of the old functional operculum to its own former state ; a reversal of asymmetry is thus induced.

When the body of Hydroides is cut into two in the thoracic region, in some cases a specimen with two symmetrical opercula is produced, but. in most cases one operculum is bigger than the other. Since, however, either the right or the left is able to develop into the functional organ, the inhibitory influence would act at first in both alike, unless a threshold value is assumed. Then it is only a question of chance which one gets the start of the other. (In Hydroides norvegica it is as a rule the right-hand one that grows more quickly than the other.) When this chance is missed either both rudiments develop into two functional organs, one as large as the other, or both remain rudimentary. I. Irosa (1921) has described a specimen of Hydroides pectinata (H. norvegica according to P. Fauvel, 1927, p. 359) with two symmetrical opercula under the name of Hydroides pectinata var. bioperculata (l.c., p. 50, Tav. 4, Fig. 8). I think this is a 
case of double opercular formation in nature instead of one in the ordinary case.

As a rule the anterior regeneration of Hydroides is only possible from the thoracic segments and not from the abdominal ones; the power of anterior regeneration is, therefore, quite limited in this Polychæte. Moreover there is generally no recovery of the missing setigerous segments, only the head being regenerated. According to Zeleny (1905), the most posterior region showing regeneration of branchial and opercular structures (in Hydroides uncinata) is an anterior cut surface, located between the next to the last (6th) and the last (7th) thoracic segments.

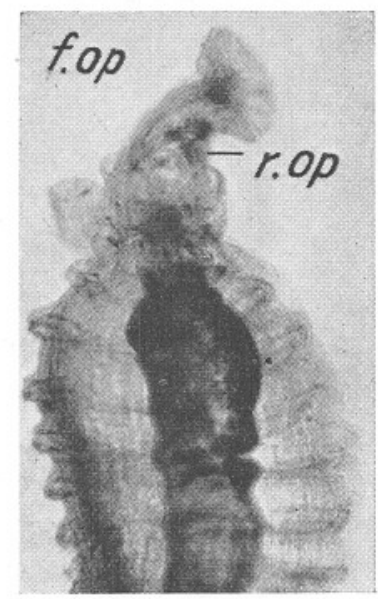

FIG. 5.-Anterior regeneration from abdominal segment.

In a cut posterior to this limit the anterior cut surface heals over, but no regeneration takes place.

Contrary to this general rule, I have got a specimen in which a head is regenerated from one of the abdominal segments. Figure 5 is a photographic reproduction of this specimen. As is well shown by the figure, there are no specialised thoracic segments, while the head is formed directly in front of the abdominal segments. Since there is no recovery of the setigerous segments in this Polychæte, there is little difficulty in concluding that regeneration can take place from one of the abdominal segments. Actually I found this specimen in a lot, in which the experimental animals. were divided into two by a transverse section at the anterior part of the abdominal region. Besides this peculiarity of the exceptional regeneration, the most important thing which should not be overlooked, is that its two opercula are not symmetrical in development, the right-handed (left side in the figure) being far larger than the left. That in Hydroides 
norvegica the opercula show an asymmetrical development from the beginning, is here again confirmed. This tendency to asymmetry, according to Zeleny's observation (l.c., p. 41), was already distinct in the larval stage, when the opercula first differentiate. Therefore, it may be said that Hydroides opercula have a sort of labile asymmetry from the beginning of their development. However, there is no critical stage when it is invariably fixed. It is easily reversed to another type of asymmetry or is altered to the symmetrical condition by an external influence. In this respect the nature of the asymmetry seems to be quite different from that of the male fiddler crab, in which the asymmetry of the chelæ is said to be derived from the symmetrical condition relatively late in development, and the relation, once attained, becomes fixed and there is no more reversal of the asymmetry.

\section{SUMMARY.}

(1) The asymmetrical development in Hydroides opercula is due to the growth difference between two sides in the same kind of organs. The presence of a functional organ holds its mate in check. Therefore, taking off the functional operculum results in the development of the rudimentary side.

(2) This phenomenon of compensatory regulation cannot be considered to be brought about by a nervous reflex set in motion by the stimulation of damaged nerves in the operated operculum, since, for example, the inhibitory influence to a certain extent depends on the degrees of injury.

(3) It is also not proved to be due to the production of a certain chemical substance which stimulates the rudimentary organ to grow, while the wound itself affects directly the inhibitory action of the functional operculum.

(4) The inhibitory influence is only localized in the operculum and not outside of it. Therefore, cutting off the gill filaments or other parts of the body does not bring a reversal of asymmetry.

(5) Within the limit of the operculum there is no special centre of the inhibitory influence, which depends on the intactness of the internal constitution of the organ. Those peripheral parts outside the limit of direct blood supply can be cut off, without the resulting reversal of asymmetry. A simple puncturing with a fine needle also has no effect. But greater injury accompanied by an outflow of blood always starts the rudimentary organ developing. 
(6) The functional operculum has no regenerative capacity, while the rudimentary one still has. The injured operculum is thrown off sooner or later from the body, and a new start repeats from the beginning the original development. By accelerating this regenerative development of the old functional organ, or properly controlling the growth of the rudimentary organ on the opposite side, a symmetrical animal with two large opercula can easily be derived. On the other hand, if both rudiments antagonistically check each other's development, a symmetrical animal with two small rudimentary opercula is produced.

(7) Since, however, either the right or the left or even both rudiments can be made to develop into a functional organ, the inhibitory influence must act at first on both alike, unless a threshold value is assumed. In the present species of Polychæte it is as a rule the right-handed one that grows more quickly than the other.

(8) With regard to the nature of the asymmetry in Hydroides it seems to be quite different from that in the male Gelasimus in which the asymmetry of the chelæ is said to be derived from the symmetrical condition relatively late in development, and the relation, once attained, becomes fixed.

(9) In Hydroides norvegica at least, the opercula show a tendency to right-handed growth from the beginning of their development, but this condition of asymmetry is very unstable and is easily reversible by an external influence even in the adult state, i.e. there is no critical stage at which it is irreversibly determined.

\section{REFERENCES.}

Baudouin, M. 1903. Autotomie et Repousse des Pinces chez Gelasimus. Bull. Mus. Hist. Nat., Paris, 9, p. 341.

Irosa, I. 1921. Revisione dei Serpulidi e Sabellidi del Golfo di Napoli. Pub. Stat. Zool. Napoli, 3, p. 47.

Morgan, T. H. 1923. Development of Asymmetry in the Fiddler Crab. Amer. Nat., 5\%, p. 269.

Morgan, T. H. 1924. Artificial Induction of Symmetrical Claws in Male Fiddler Crabs. Amer. Nat., 58, p. 289.

Przibram, H. 1901. Experimentelle Studien über Regeneration. Roux' Arch., 11, p. 321.

Przibram, H. 1907. Scheerenumkehr bei Dekapoden. Roux' Arch., 25, p. 266. 
Przibram, H. 1907. Experimental-Zoologie. II.

WiLson, E. B. 1903. Reversal Asymmetry in the Regeneration of the Chelæ in Alpheus heterochelis. Biol. Bull., 4, p. 197.

Zeleny, C. 1901. A case of Compensatory Regulation in the Regeneration of Hydroides dianthus. Roux' Arch., 13, p. 597.

Zeleny, C. 1905. Compensatory Regulation. J. Exp. Zool., 2 , p. 1. 